\title{
Dynamic Model of the Axially Moving Viscoelastic Belt System with Tensioner Pulley
}

\author{
Yanqi Liu ${ }^{1, a}$, Hongyu Wang ${ }^{2, b}$, Dongxing $\mathrm{CaO}^{3, \mathrm{c}}$, Xiaoling Gai, d \\ ${ }^{1}$ Beijing Key Laboratory of Environment Noise and Vibration, Beijing Municipal Institute of Labour \\ Protection, Beijing, 100054, China \\ ${ }^{2}$ State Key Laboratory of Tribology, Tsinghua University, Beijing, 100084, China \\ ${ }^{3}$ College of Mechanical Engineering and Applied electronics, Beijing University of Technology, \\ Beijing, 100124, China \\ aemail: jdyqliu@163.com, 'email: axial888@163.com, 'email: caostar@bjut.edu.cn, ${ }^{d} e m a i l:$ \\ gxldynmg@163.com
}

Keywords: Moving Belt; Nonlinear vibration; Dynamical model; Viscoelastic

\begin{abstract}
Dynamic model of the moving belt system with tensioner pulley is studied in this paper. Belt is considered elastic continuous element, and pulley and tensioner arm are discrete element. Considering the geometrical nonlinearity and Kelvin viscoelastic constitutive model, the kinetic energy, potential energy and work done by external forces of moving belt system with tensioner pulley are given respectively. Then, the nonlinear equations of motion for the moving viscoelastic belt system with tensioner pulley are obtained by using the Hamilton's principle. Note that nonlinear oscillations of the belt and pulley oscillations are coupled.
\end{abstract}

\section{Introduction}

The moving belt system with tensioner pulley has wide applications in the areas of engineering. As an axially moving material, the transverse vibration of moving belt has been investigated extensively [1-3]. For moving belt system with tensioner pulley, oscillations of the belt and pulley oscillations are coupled and it exhibit complex dynamical equation and dynamical characteristics. However, the most studies neglect the viscoelasticity of the belt and regard belt as elastic material.

In this paper, considering the viscoelasticity of belt material, nonlinear equations of motion of moving belt system with tensioner pulley are derived. Oscillations of the belt and pulley oscillations are coupled and the dynamical equations are complex.

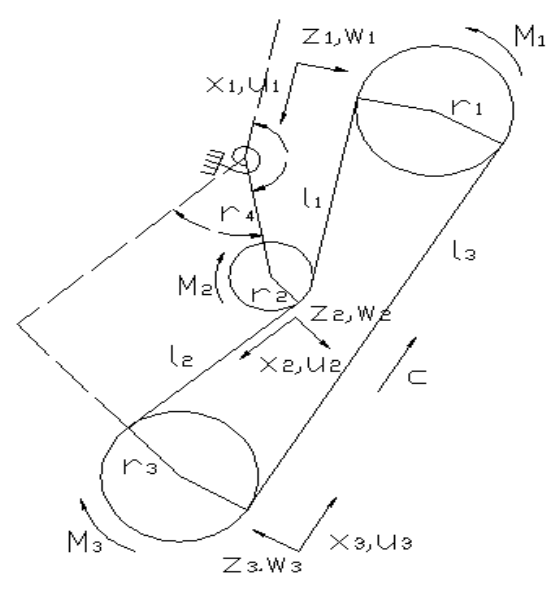

Fig.1 Three-pulley drive system with tension pulley 


\section{Dynamic Model of the Moving Belt System with Tensioner Pulley}

In this paper, it is assumed that the belt tension dominates the transverse stiffness of the belt. Then, an axially moving viscoelastic belt can be modeled as a string, and the pulleys are considered discrete element, as shown in Fig.1.

For string model, the effects of the moment of inertia of the cross-sectional area and shear deformation can be ignored. The strain of string caused by axial displacement is given by

$$
\varepsilon_{i}=u_{i, x}+\frac{1}{2} w_{i, x}^{2}(i=1,2,3)
$$

here, $u_{i}$ and $w_{i}$ are the longitudinal and transverse deflections in span $i$ from equilibrium.

The belt is considered homogeneous viscoelastic material and it obeys the linear viscoelastic differential constitutive relation. Taking into account the Kelvin viscoelastic constitutive model, the dynamic tensions in the belt spans become

$$
p_{d i}=E A\left(u_{i, x}+\frac{1}{2} w_{i, x}^{2}\right)+\eta \frac{\partial\left(u_{i, x}+\frac{1}{2} w_{i, x}^{2}\right)}{\partial t} \quad(i=1,2,3)
$$

where, $\mathrm{E}$ is the stiffness constant of belt, $\eta$ is the dynamical viscous coefficient of belt.

In the following analysis, the extended Hamilton's principle is used to derive the nonlinear governing equations of motion of the belt and pulley coupled system.

The kinetic energy, $\mathrm{T}$, of the moving viscoelastic belt and pulley coupled system is

$$
\begin{aligned}
& T=\frac{1}{2} J_{1}\left(\frac{\mathrm{c}}{r_{1}}+\theta_{1, t}\right)^{2}+\int_{0}^{L_{1}} \frac{1}{2} m\left[\left(w_{1, t}+c w_{1, x}\right)^{2}+\left(u_{1, t}+c u_{1, x}+c\right)^{2}\right] d x_{1} \\
& +\frac{1}{2} J_{2}\left(\frac{\mathrm{c}}{r_{2}}+\theta_{2, t}\right)^{2}+\int_{0}^{L_{2}} \frac{1}{2} m\left[\left(w_{2, t}+c w_{2, x}\right)^{2}+\left(u_{2, t}+c u_{2, x}+c\right)^{2}\right] d x_{2} \\
& +\int_{0}^{L_{3}} \frac{1}{2} m\left[\left(w_{3, t}+c w_{3, x}\right)^{2}+\left(u_{3, t}+c u_{3, x}+c\right)^{2}\right] d x_{3}+\frac{1}{2} J_{3} \theta_{3, t}^{2}+\frac{1}{2} J_{4}\left(\frac{\mathrm{c}}{r_{4}}+\theta_{4, t}\right)^{2}
\end{aligned}
$$

where $J_{i}, r_{i}$ and $\theta_{i}$ are the mass moment of inertia, radius, and rotation angle from equilibrium of the ith discrete element, respectively. $\mathrm{m}$ is mass of belt, $\mathrm{c}$ is the belt axial velocity, and $L_{i}$ is the length of belt $\operatorname{span}_{i}$. Subscripts ${ }_{, x}$ or ${ }_{, t}$ denote the partial derivative with respect to that variable, $\mathrm{x}$ represents a spanwise coordinate, and $\mathrm{t}$ is time.

The potential energy of the belt and pulley coupled system is

$$
\begin{aligned}
& U=\frac{1}{2} K_{r}\left(\theta_{3}+\theta_{3 r}\right)^{2}+\frac{E A}{2} \int_{0}^{\phi_{1}}\left(\frac{P_{3}\left(l_{3}\right)}{E A}+\frac{\sigma_{1}}{\phi_{1}}\left(\frac{P_{1}(0)}{E A}-\frac{P_{3}\left(l_{3}\right)}{E A}\right)\right)^{2} r_{1} d \sigma_{1} \\
& +\frac{E A}{2} \int_{0}^{L_{1}}\left(\frac{P_{01}}{E A}+u_{1, x}+\frac{1}{2} w_{1, x}^{2}\right)^{2} d x_{1}+\frac{E A}{2} \int_{0}^{\phi_{2}}\left(\frac{P_{1}\left(l_{1}\right)}{E A}+\frac{\sigma_{2}}{\phi_{2}}\left(\frac{P_{2}(0)}{E A}-\frac{P_{1}\left(l_{1}\right)}{E A}\right)\right)^{2} r_{2} d \sigma_{2} \\
& +\frac{E A}{2} \int_{0}^{L_{2}}\left(\frac{P_{02}}{E A}+u_{2, x}+\frac{1}{2} w_{2, x}^{2}\right)^{2} d x_{2}+\frac{E A}{2} \int_{0}^{\phi_{4}}\left(\frac{P_{2}\left(l_{2}\right)}{E A}+\frac{\sigma_{4}}{\phi_{4}}\left(\frac{P_{3}(0)}{E A}-\frac{P_{2}\left(l_{2}\right)}{E A}\right)\right)^{2} r_{2} d \sigma_{2} \\
& +\frac{E A}{2} \int_{0}^{L_{3}}\left(\frac{P_{03}}{E A}+u_{3, x}+\frac{1}{2} w_{3, x}^{2}\right)^{2} d x_{3}
\end{aligned}
$$

where $\sigma_{\mathrm{i}}$ and $\phi_{\mathrm{i}}$ are the coordinate and wrap angles on pulley i respectively, and $\theta_{3 \mathrm{r}}$ is the tensioner spring deflection in the reference position. Here the total tension of belt span $\mathrm{i}$ is 


$$
P_{i}=P_{t i}+P_{d i}=P_{t i}+E A\left(u_{i, X}+\frac{1}{2} w_{i, X}^{2}\right)+\eta \frac{\partial}{\partial t}\left(u_{i, X}+\frac{1}{2} w_{i, X}^{2}\right)
$$

The work done by external forces includes that done by the tractive tension $P_{t i}$ and viscoelastic damping. The expression for external work is

$$
W=\sum_{i=1}^{3} P_{t i} \varepsilon_{i}+\sum_{i=1}^{3} \eta \frac{\partial \varepsilon_{i}}{\partial t}
$$

According to the geometrical nonlinearity and Kelvin viscoelastic constitutive model, the kinetic energy, potential energy and work of the moving viscoelastic belt system with tensioner pulley are given respectively.

\section{Equations of Motion of Moving Viscoelastic Belt System with Tensioner Pulley}

In this section, the equations of motion of the moving belt and pulley will be obtained by Hamilton’s principle. Substituting equations (3), (4) and (6) into Hamilton’s principle,

$$
\int_{t_{1}}^{t_{2}}(\delta T-\delta U+\delta W) d t=0
$$

Here T, $U$ and $\delta W$ are the kinetic energy, the potential energy, and the virtual work done by the external forces, respectively. After integrating by parts where needed, the government equations of motion for the belt spans are

$$
\begin{aligned}
& m u_{i, t t}+2 m c u_{i, x t}+m c^{2} u_{i, x x}=\left(P_{d i}+P_{t i}\right)_{, x} \quad(i=1,2,3) \\
& m w_{i, t t}+2 m c w_{i, x t}+m c^{2} w_{i, x x}=\left[\left(P_{d i}+P_{t i}\right) w_{i, x}\right]_{, x} \quad(i=1,2,3)
\end{aligned}
$$

Note that each field equation has three acceleration terms: the local acceleration $w_{i, t t}$ or $u_{i, t}$, the Coriols acceleration $2 c w_{i, x t}$ or $2 c u_{i, x t}$ and the centripetal acceleration $c^{2} w_{i, x x}$ or $c^{2} u_{i, x x}$.

Owing to longitudinal vibration compared to transverse vibration is very small, as a consequence, one may neglect the longitudinal vibration. Under the "quasi-static stretching" assumption, the dynamic tension in the belt span $P_{d i}$ become

$$
p_{d i}=\frac{E A}{L_{i}}\left(u_{i}\left(L_{i}, t\right)-u_{i}(0, t)+\frac{1}{2} \int_{0}^{L_{i}} w_{i, x}^{2} d x_{i}\right)+\eta \frac{\partial\left(u_{i}\left(L_{i}, t\right)-u_{i}(0, t)+\frac{1}{2} \int_{0}^{L_{i}} w_{i, x}^{2} d x_{i}\right)}{\partial t}
$$

and are uniform throughout the span. With this assumption, the equations for the transverse vibration of moving belt reduce to

$$
m w_{i, t t}+2 m c w_{i, x t}+m c^{2} w_{i, x x}=\left(P_{d i}+P_{t i}\right) w_{i, x x} \quad(i=1,2,3)
$$

The equations of motion for the discrete elements are obtained as follows:

For pulley 1

$$
\left(\mathrm{P}_{\mathrm{d} 1}-\mathrm{P}_{\mathrm{d} 3}\right) \mathrm{r}_{1}+\left(P_{o 1}-P_{o 3}\right) \mathrm{r}_{1}=J_{1} \theta_{1, t t}
$$

For pulley2

$$
\left(\mathrm{P}_{\mathrm{d} 2}-\mathrm{P}_{\mathrm{d} 1}\right) r_{2}+\left(P_{o 2}-P_{o 1}\right) r_{2}=J_{2} \theta_{2, t t}
$$

For pulley 3

$$
\left(\mathrm{P}_{\mathrm{d} 3}-\mathrm{P}_{\mathrm{d} 2}\right) r_{4}+\left(P_{o 3}-P_{o 2}\right) r_{4}=J_{4} \theta_{4, t t}
$$

For the tensioner arm 


$$
\begin{aligned}
& {\left[m c W_{1, t}\left(I_{1}\right)+m c^{2}-\left(P_{d 1}+P_{o 1}\right) W_{1, x}\left(I_{1}\right)\right] r_{4} \cos \left(\theta_{4}+\alpha_{1}\right)} \\
& +\left(m c^{2}-\left(P_{d 1}+P_{o 1}\right)\right) r_{4} \sin \left(\theta_{4}+\alpha_{1}\right) \\
& +\left[m c w_{2, t}(0)-\left(P_{d 2}+P_{o 2}-m c^{2}\right) W_{2, x}(0)\right] r_{4} \cos \left(-\theta_{4}+\alpha_{2}\right) \\
& +\left(P_{d 2}+P_{o 2}-m c^{2}\right) r_{4} \sin \left(-\theta_{4}+\alpha_{2}\right)+M_{4}-k_{r}\left(\theta_{4}+\theta_{4 r}\right)=J_{4} \theta_{4, t t}
\end{aligned}
$$

For ease, the motion equations of the discrete elements in terms of displacements along the arc-lengths are

$$
\begin{aligned}
& m w_{, t t}+2 m c w_{, x t}+m c^{2} w_{, x x}=\left(P_{d i}+P_{t i}\right) w_{, x x} \quad(i=1,2,3) \\
& m_{1} \chi_{1, t t}+\left(k_{1}+k_{3}\right) \chi_{1}-k_{1} \chi_{2}-k_{3} \chi_{3}-k_{1} \cos \varphi_{1} \chi_{4}+\left(\frac{\eta}{L_{1}}+\frac{\eta}{L_{3}}\right) \chi_{1, t}-\frac{\eta}{L_{1}} \chi_{2, t}-\frac{\eta}{L_{3}} \chi_{3, t} \\
& -\frac{\eta}{L_{1}} \cos \varphi_{1} \chi_{4, t}=P_{d 1 N L}-P_{d 3 N L} \\
& m_{2} \chi_{2, t t}-k_{1} \chi_{1}+\left(k_{1}+k_{2}\right) \chi_{2}-k_{2} \chi_{3}+\left(k_{1} \cos \varphi_{1}-k_{2} \cos \varphi_{2}\right) \chi_{4}-\frac{\eta}{L_{1}} \chi_{1, t}+\left(\frac{\eta}{L_{1}}+\frac{\eta}{L_{2}}\right) \chi_{2, t} \\
& -\frac{\eta}{L_{2}} \chi_{3, t}+\left(\frac{\eta}{L_{1}} \cos \varphi_{1}-\frac{\eta}{L_{2}} \cos \varphi_{2}\right) \chi_{4, t}=P_{d 2 N L}-P_{d 1 N L} \\
& m_{3} \chi_{3, t}-k_{3} \chi_{1}-k_{2} \chi_{2}+\left(k_{2}+k_{3}\right) \chi_{3}+k_{2} \chi_{4} \cos \varphi_{2}-\frac{\eta}{L_{3}} \chi_{1, t}-\frac{\eta}{L_{2}} \chi_{2, t}+\left(\frac{\eta}{L_{3}}+\frac{\eta}{L_{2}}\right) \chi_{3, t} \\
& +\frac{\eta}{L_{2}} \cos \varphi_{2} \chi_{4, t}=P_{d 3 N L}-P_{d 2 N L} \\
& m_{4} \chi_{4, t t}-\left[m c w_{1, t}\left(l_{1}\right)-P_{t 1} w_{1, x}\left(l_{1}\right)\right] \cos \left(\theta_{4}+\alpha_{1}\right)-\left[m c w_{2, t}(0)-P_{t 2} w_{2, x}(0)\right] \cos \left(-\theta_{4}+\alpha_{2}\right) \\
& +\left(k_{1}\left(\chi_{4} \cos \varphi_{1}+\chi_{2}-\chi_{1}\right)+\frac{\eta}{L_{1}}\left(\chi_{4, t} \cos \varphi_{1}+\chi_{2, t}-\chi_{1, t}\right)\right) w_{1, x}\left(l_{1}\right) \cos \left(\theta_{4}+\alpha_{1}\right) \\
& +\left(k_{2}\left(\chi_{3}-\chi_{2}+\chi_{4} \cos \varphi_{2}\right)+\frac{\eta}{L_{2}}\left(\chi_{3, t}-\chi_{2, t}+\chi_{4, t} \cos \varphi_{2}\right)\right) w_{2, x}(0) \cos \left(-\theta_{4}+\alpha_{2}\right) \\
& -k_{r}\left(\chi_{4}+\frac{\theta_{4 r}}{r_{4}}\right)=-P_{d 1 N L} w_{1, x}\left(l_{1}\right) \cos \left(\theta_{4}+\alpha_{1}\right)-P_{d 2 N L} w_{2, x}(0) \cos \left(-\theta_{4}+\alpha_{2}\right) \\
& \text { where } m_{i}=J_{i} / r_{i}^{2}, \chi_{i}=r_{i} \theta_{i} \text {. }
\end{aligned}
$$

Equation (13a)-(13e) are the nonlinear equations of motion for the moving viscoelastic belt system with tensioner pulley. They represent a set of partial and ordinary differential equations describing the coupled transverse vibration of the moving belts and the rotational motions of the pulleys.

\section{Conclusion}

The system of moving viscoelastic belt with tensioner pulley is divided elastic continuous element, belt, and discrete element, pulley and tensioner arm. Considering Kelvin viscoelastic constitutive model, the kinetic energy, potential energy and work done by external forces of moving belt system with tensioner pulley are given respectively. Then, the nonlinear equations of motion of the viscoelastic moving belt system with tensioner pulley are derived by using the Hamilton's principle. Note that nonlinear oscillations of belt and pulley oscillations are coupled.

\section{Acknowledgement}

The research is supported by Natural Science Foundation of China (11102093,11272016), 
Natural Science Foundation of Beijing (Z2001015201202) and Youth Backbone Foundation of Beijing Academy of Science and Technology.

\section{References}

[1] L.Zhang, J.W.Zu. One-to-one auto-parametric resonance in serpentine belt drive systems [J]. Journal of Sound and Vibration, 2000 232(4) 783-806.

[2] R.G. Parker. Efficient eigensolution,dynamic response,and eigensensitivity of serpentine belt drives [J]. Journal of Sound and Vibration, 2004270 15-38.

[3] L. Kong, R. G. Parker. Equilibrium and belt-pulley vibration coupling in serpentine belt drives [J]. Journal of Applied Mechanics, 2003 70(5) 739-750. 